\title{
The Importance of Contrast Enhancement in Medical Images Analysis and Diagnosis
}

\author{
Dr. Faten A. Dawood \\ Department of Computer Science \\ College of Science, University of Baghdad \\ Baghdad, Iraq
}

\author{
Dr. Ziad M. Abood \\ Department of Physics \\ College of Education, Mustansiriyah University \\ Baghdad, Iraq
}

\begin{abstract}
Contrast enhancement is a significant factor in any subjective evaluation of image quality which used to enhance the overall quality of the medical image for feature visualization and clinical measurement. This study presents a number of contrast enhancement techniques for medical images analysis. These techniques were applied on different type of medical images such as: MRI, CT-Scan and X-ray to improve image quality and come up with an acceptable image contrast. The proposed method included different enhancement techniques: logarithm and Exponential equations was created to improve the illumination and contrast of medical images, Image quality coefficients were extracted and compared with image quality coefficients for the same images, which were processed by the modified filter, and showed that the proposed method gave better results.
\end{abstract}

Keywords-Medical Images; MRI; CT-Scan; X-ray; Contrast enhancement

\section{INTRODUCTION}

The aim of this study is to improve the image quality, enhance features and gain better characteristics of several medical images for a precise analysis and diagnosis. Medical imaging is one of the most advanced field of imaging that required for creating a visual representation of the internal structure of the human body so as to diagnose any abnormalities or diseases. Recent advances in medical image modalities, including Magnetic Resonance Imaging (MRI), Computed Tomography (CT), X-ray-based devices, Ultrasound (US) and Positron Emission Tomography (PET) [1]. Medical image analysis is a particularly difficult problem due to the inherent characteristics of these images in being low contrast, containing speckle noise, having signal dropouts and complex anatomical structures [2]. Therefore, it is very important to enhance the contrast of such images before further processing and analysis.

Contrast enhancement is a significant factor in any subjective evaluation of image quality which used to enhance the overall quality of the medical image for feature visualization and clinical measurement. Nowadays, most of contrast enhancement techniques have been applied to improve the visual appearance by increasing dominance of some features or by reducing ambiguity between different regions of the image[3,4].Several techniques are existing to improve the contrast and brightness of an image. The histogram modification techniques have been widely used for enhancing the contrast of medical image. The histogram equalization (HE) is one of the most popular technique which frequently used due to its simplicity and explicitness $[5,6]$. There are several methods have been applied to determine the level of contrast enhancement that carried out through modifications on the HE [7].Adaptive Histogram Equalization (AHE) provides a local contrast enhancement which computes the histogram of a local window centered at a given pixel [8].Therefore, all regions that having different gray scale ranges can be enhanced simultaneously. A generalization of AHE is Contrast Limiting AHE (CLAHE) which has more flexibility in choosing the local histogram mapping function. Undesired noise can be reduced by selecting the clipping level of the histogram [9].

Another method for medical image enhancement is Gamma correction [10].In this method, Gamma values of individual pixels are locally optimized by minimizing the homogeneity of co-occurrence matrix of the original image. The Gamma correction method improves the contrast of image by enhancing the dynamic range [11]. A review of most popular contrast enhancement techniques for medical images can be found in $[12,13]$.

\section{MEDICAL IMAGING MODALITIES}

Several medical imaging modalities have been used for analyzing anatomical structures such as bones, muscles, blood vessels, tissue types, pathological regions such as cancer, multiple sclerosis lesions [14-16]. Therefore, this study focuses on the importance of contrast enhancement in medical images for precise analysis and diagnosis. Here, three medical imaging modalities will be discussed; MRI, CT-scan and X-ray such as shown in Fig. 1. Each one has its own mechanism of providing relevant physiological information of the organ being imaged.

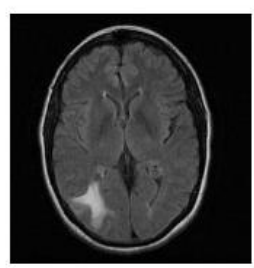

(a) Brain MRI

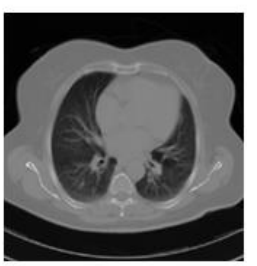

(b) A Chest CI

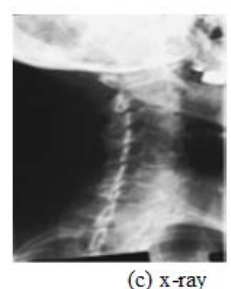

(c) $x$-ray
Fig. 1. Examples of medical imaging modalities. 


\section{A. Magnetic Resonance Imaging}

Magnetic resonance imaging (MRI) is a medical imaging modality that is based on the same principals of nuclear magnetic resonance spectroscopy. MRI is the most powerful and noninvasive tool for clinical diagnosis of diseases [17]. The MRI scanner uses magnetic and radio waves to create pictures of tissues, organs and other structures within the body. The images that produced by an MRI scan are better in displaying good details. Therefore, it is possible to pictures all the tissue in the body by using an MRI scanner. These tissue such as bones (has the least hydrogen atoms) becomes dark, while the fatty tissue (that has many hydrogen atoms) looks much brighter. It is possible to gain information about the different types of tissues that are present by changing the timing of the radio wave pulses [18].

\section{B. $\quad$ Computed Tomography}

Computed Tomography (CT) is a technique which utilizes X-rays in conjunction with computing algorithms to image tissues in the body. CT is one of the important diagnostic tool in the field of medical imaging which used to image the internal structure of human body. It provides good contrast amongst of different soft tissues of the body which especially make it useful for imaging the muscles, brain and cancers compared with other medical imaging techniques. Some advancement of CT machines technology have been introduced to increase the contrast of the CT images which are being used for diagnostic purposes [19, 20].

\section{X-ray}

$\mathrm{X}$-ray images are being used to image the internal structure of human body. In the field of medicine, it is one of the most widely used diagnostic tools. X-Ray is used for capturing images of the internal body structure that help the radiologists in recognizing the internal problems. It is the most useful imaging modality to check for the bone fractures. Although of several advantages of X-Ray technology, but it generates low contrast images. The main reason for low contrast of X-ray images due to presence of amount liquid in human body. One can increase the power of X-Rays for capturing images but it may harm human body/ bones [3].

\section{CONTRAST ENHANCEMENT TECHNIQUES}

Contrast enhancement techniques have various application fields especially in medical imaging for enhancing visual quality of low contrast image. It is a required step in medical image analysis for highlighting the important features or the features that are not properly visible. The goal of most contrast enhancement techniques is to determine an optimal transformation function relating original gray level and the displayed intensity such that contrast between adjacent structures in an image.

\section{Histogram Equalization}

Histogram equalization (HE) is a very popular technique and widely used for enhancing the contrast of an image. Its basic idea lies on mapping the gray levels based on the probability distribution of the input gray levels. HE improves contrast by obtaining a uniform histogram and can be used on a whole image or just on a part of an image. This technique attempts to spread out the gray levels in an image and reassigns the brightness value of pixels based on the image histogram. Histogram equalization technique is effective only when the original image has low contrast to start with, otherwise histogram equalization may degrade the image quality [5].

\section{E. Adaptive Histogram Equalization}

Adaptive Histogram Equalization (AHE) is a technique by using localized histogram equalization which considers a local window for each individual pixel and computes the new intensity value based on the local histogram defined in the local window. Adaptive characteristics can give better result, but it computation is hard enough even though there are some fast techniques for updating the local histograms [21]. In this method, the contrast of the image is enhanced by transforming the values in the intensity image. AHE attempts to overcome the limitations of global histogram equalization by providing the desired information in a single image. Thus, this approach is more popular and effective for contrast enhancement of the grayscale and color images.

\section{F. $\quad$ Contrast Limited Adaptive Histogram Equalization}

Contrast limited adaptive histogram (CLAHE) is a technique that is used for improving the local contrast of images. It is a combination of $\mathrm{HE}$ and adaptive histogram equalization, the histogram is equalized in blocks with a predefined clip limit in an adaptive manner. CLAHE does not operate on the whole image but it works on small regions of the images, named tiles. On each tile it works like ordinary Histogram Equalization (HE) [22].

\section{IMAGE QUALITY STATISTICS}

The following statistics were applied to measure the image quality applied by the present study filters:

\section{G. $\quad$ Mean Squared Error}

Mean Squared Error (MSE) known as the cumulative square error between the resulting image and the original image, and calculated from the following relationship:[23]

$$
M S E=\frac{1}{M N} \sum_{y=1}^{N} \sum_{x=1}^{M}\left[I_{1}(x, y)-I_{2}(x, y)\right]^{2}
$$

Where $I_{l}(x, y)$ is the original image element in the location $(x, y) . I_{2}(x, y)$ is the image element that required account in the location $(x, y) . \mathrm{M}, \mathrm{N}$ : size of image. 


\section{A. Peak Signal to Noise Ratio}

The peak signal-to-noise ratio (PSNR) means the highest value, the lowest value to signal noise: [24]

$$
P S N R=10 \log \left(\frac{(L-1)^{2}}{M S E}\right)
$$

Where L: gray-level numbers. PSNR is measured with unite decibel $(\mathrm{dB})$.

\section{B. Root Mean Square Error}

Root Mean Square Error (RMSE) is defined as the square root of the error rate of the MSE. The lower the value of the error: [25]

$$
R M S E=\sqrt{\frac{1}{M N} \sum_{y=1}^{N} \sum_{x=1}^{M}\left[I_{1}(x, y)-I_{2}(x, y)\right]^{2}}
$$

\section{THE PROPOSED METHOD}

In the present study, contrast enhancement is an important pre-processing step in medical images for a right analysis and diagnosis. Therefore, the following equation was developed, consisting of the logarithmic and exponential function:

$$
\mathrm{C}=0.5\left[\log (\mathrm{r})^{\mathrm{g}}+\exp (\mathrm{r})\right]
$$

Where C: contrast Enhancement, r: pixel intensity (0-255), and $\mathrm{g}$ values taken from Fig. 2.

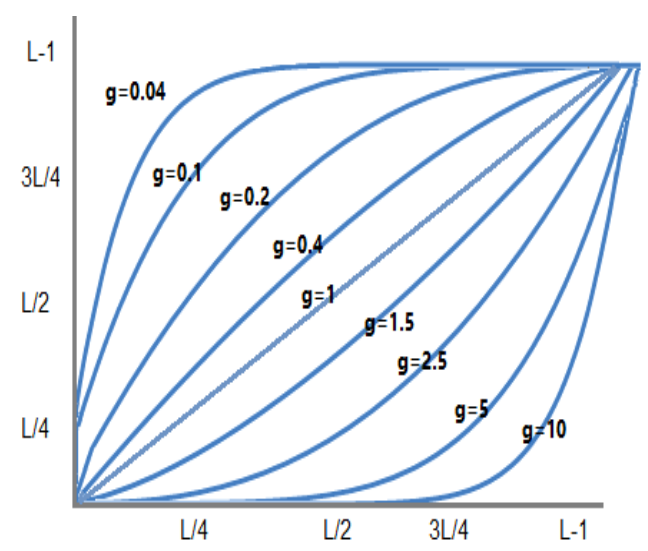

Fig. 1. Relationship between the input image intensity (r) and the enhanced image intensity (C) calculated from equation (4) [26].

Then, the contrast of the image can be enhanced through a 3 x 3 mask convolution. Fig. 3 shows the block diagram including main steps of the proposed method that applied in this present study.

After the text edit has been completed, the paper is ready for the template. Duplicate the template file by using the Save As command, and use the naming convention prescribed by your conference for the name of your paper. In this newly created file, highlight all of the contents and import your prepared text file. You are now ready to style your paper; use the scroll down window on the left of the MS Word Formatting toolbar.

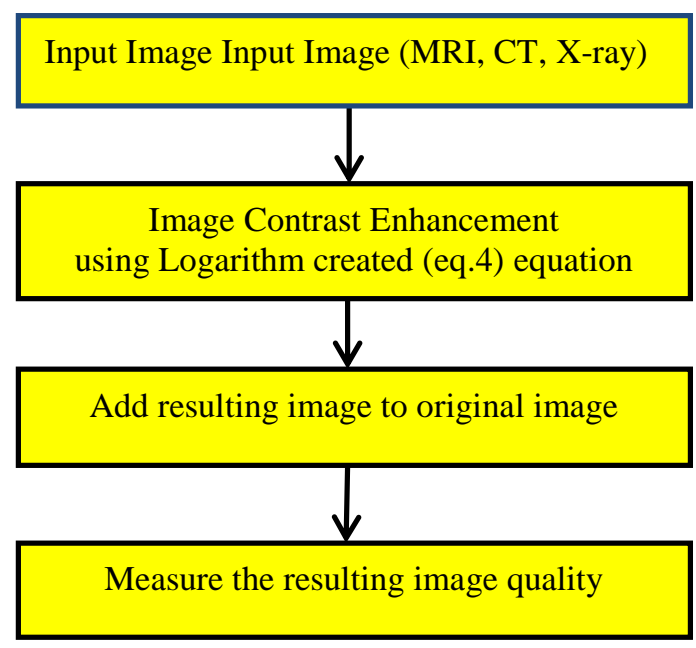

Fig. 2. Block diagram of the proposed method.

\section{EXPERIMENTAL RESULTS AND DISCUSSION}

The proposed method has been implemented on original medical images of CT-scan, MRI and X-ray. Fig. 4 shows the experimental results of proposed method by applying the $3 \times 3$ mask according to equation (4) on the original images. It can be noted by visual observation, the overall image quality has been improved as well as the contrast of each image has been enhanced.

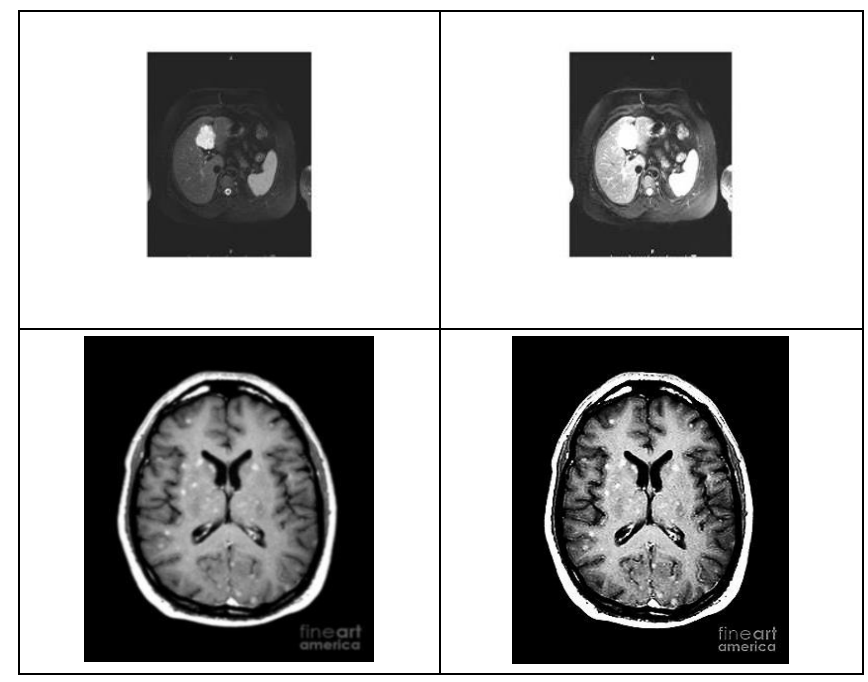




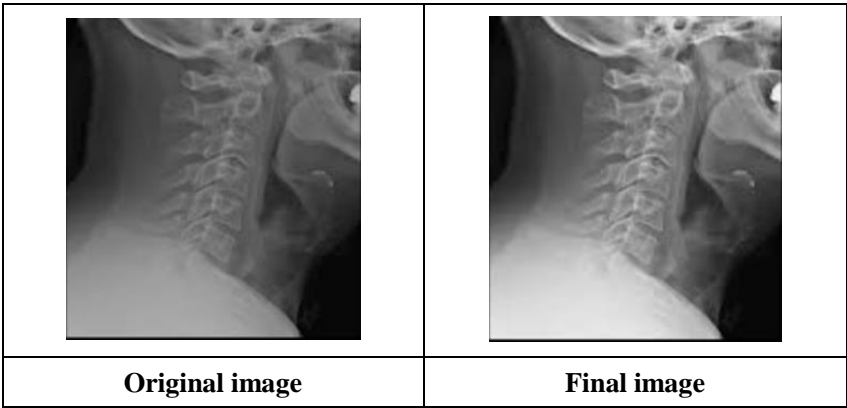

Fig. 3. The medical images before and after contrast enhancement by applied equation (4) and add to original image.

In order to measure the resultant quality of enhanced images (such as shown in Fig. 3), three statistics metrics including: PSNR, MSE, and RMSE have been used. Table I presents the values of these metrics that performed for the enhanced medical images.

TABLE I. PSNR, MSE, AND RMSE VALUES FOR ENHANCED IMAGES USING THE CREATED EQUATION

\begin{tabular}{|c|c|c|c|}
\hline \multirow{2}{*}{} & \multicolumn{3}{|c|}{ Image Quality Statistics } \\
\cline { 2 - 4 } MRI & PSNR & MSE & RMSE \\
\hline CT & 45.12 & 2.54 & 4.23 \\
\hline x-ray & 33.78 & 2.41 & 15.14 \\
\hline
\end{tabular}

\section{CONCLUSION}

The created equation (Exponential and logarithm) is enhancement of illumination and contrast of medical images with good results, and the equation that was first introduced and applied to digital images. The statistical results of PSNR, MSE and RMSE showed the efficiency of the equation (equation 4) in improving the illumination and contrast of medical images, especially for CT images.

\section{REFERENCES}

[1] Sonka, M., Hlavac, V., \& Boyle, R. (2014). "Image processing, analysis, and machine vision”. Cengage Learning.

[2] Dawood, F. A. A., Rahmat, R. W., Dimon, M. Z., Nurliyana, L., \&Kadiman, S. B. (2011). Automatic boundary detection of wall motion in two-dimensional echocardiography images. Journal of Computer Science, 7(8), 1261-1266.

[3] Mohanta, K., \&Khanaa, V. (2013). An Efficient Contrast Enhancement of Medical X-Ray Images-Adaptive Region Growing Approach. International Journal of Engineering and Computer Science, 2(2), 208-212.

[4] El-Dahshan, E. S. A., Mohsen, H. M., Revett, K., \& Salem, A. B. M. (2014). Computer-aided diagnosis of human brain tumor through MRI: A survey and a new algorithm. Expert systems with Applications, 41(11), 5526-5545.

[5] Jagatheeswari, P., Suresh Kumar, S., \&Rajaram, M. (2009). Contrast enhancement for medical images based on histogram equalization followed by median filter.

[6] Ziad M Abood, (2013). Edges Enhancement of Medical Color Images Using Add Images, IOSR Journal of Research \& Method in Education, 2(4)

[7] Aggarwal, A., Chauhan, R. S., \&Kaur, K. (2013). An Adaptive Image Enhancement Technique Preserving Brightness Level
Using Gamma Correction. Advance in Electronic and Electric Engineering, 3(9), 1097-1108.

[8] Min, B. S., Lim, D. K., Kim, S. J., \& Lee, J. H. (2013). A novel method of determining parameters of CLAHE based on image entropy.

[9] Jin, Y., Fayad, L., \&Laine, A. (2001, July). Contrast enhancement by multiscale adaptive histogram equalization. In Proc SPIE (Vol. 4478, pp. 206-213)

[10] Amiri, S. A., \&Hassanpour, H. (2012). A preprocessing approach for image analysis using gamma correction. Department of Computer Engineering Shahrood University of Technology Iran.

[11] Hassanpour, H., Samadiani, N., \&Salehi, S. M. (2015). Using morphological transforms to enhance the contrast of medical images. The Egyptian Journal of Radiology and Nuclear Medicine, 46(2), 481-489.

[12] Thamman, P., \&Verma, A. (2014). Contrast Enhancement of Medical Images-AReview.InternationalJournal of Innovations \& Advancement in Computer Science, 3(4), 124-128.

[13] D.Nirmala (2015). Review article: Medical image contrast enhancement techniques. Journal of Chemical and Pharmaceutical Research, 7(7):1-8.

[14] Dawood, F. A., Rahmat, R. W., Kadiman, S. B., Abdullah, L. N., \&Zamrin, M. D. (2014). A hybrid method for endocardial contour extraction of right ventricle in 4-slices from 3D echocardiography dataset. Advances in bioinformatics, 2014.

[15] Bankman, I. (Ed.). (2008). Handbook of medical image processing and analysis. Academic press.

[16] Dawood, F. A., Rahmat, R. W., Kadiman, S. B., Abdullah, L. N., \&Zamrin, M. D. (2015). Measurements of mitral annular displacement in 2D echocardiography images. American Journal of Applied Sciences, 12(5), 295-303.

[17] Mansfield, P. (2004). Snapshot magnetic resonance imaging (Nobel lecture). AngewandteChemie International Edition, 43(41), 5456-5464.

[18] Tirpude, N., \&Welekar, R. (2013). Automated detection and extraction of brain tumor from MRI images. International Journal of Computer Applications, 77(4).

[19] Patel, B. C., \&Sinha, G. R. (2014). Abnormality detection and classification in computer-aided diagnosis (CAD) of breast cancer images. Journal of Medical Imaging and Health Informatics, 4(6), 881-885.

[20] Kim, S., Min, B., Oh, W., \& Lee, J. (2013). Medical image enhancement algorithm using edge-based denoising and adaptive histogramstretching. International Journal of Bio-Science and Bio-Technology, 5(5), 25-38.

[21] Pizer, S. M., Amburn, E. P., Austin, J. D., Cromartie, R. Geselowitz, A., Greer, T., \&Zuiderveld, K. (1987). Adaptive histogram equalization and its variations. Computer vision, graphics, and image processing, 39(3), 355-368.

[22] Ahmed, H. S., \&Nordin, M. J. (2011). Improving diagnostic viewing of medical images using enhancement algorithms. Journal of Computer Science, 7(12), 1831.

[23] Dawood, F. A., Rahmat, R. W., Kadiman, S. B., Abdullah, L. N., \&Zamrin, M. D. (2012). Effect comparison of speckle noise reduction filters on 2D-Echocardigraphic images. World Academy of Science, Engineering and Technology, 69(69), 425430

[24] Ziad M. Abood, Kadhim K. Kadhim, (2015), Assessment the Quality of Medical Images (CT \& MRI) by Using Wavelet Transformation (WT), International Journal of Emerging Research in Management \& Technology, vol. 4, Issue 7.

[25] Ziad M. Abood, Zahra S. Dheef\&Lamyaa M. Kadhim, 2017, Diagnosis of colon tumor tissue using fuzzy logic Vol. 12, No.1, March - IJST.

[26] Ziad M. Abood, Falah A. Bid, (2017). Enhancement the brightness and contrast of the image medical by numerical equations. Conference $23 \mathrm{~J}$. of Education College, Mustansiriyah University. 